\title{
Functional epidermal growth factor gene polymorphisms and risk of gastric cancer
}

\author{
ZHEN ZHAN $^{1 *}$, YAJUN CHEN ${ }^{2 *}$, JUAN WU $^{1}$, JUNFENG ZHANG $^{1}$, SHUJUAN TONG $^{1}$, \\ CHUNBING ZHANG $^{2}$ and YAPING YANG ${ }^{1}$
}

${ }^{1}$ Department of Chinese and Western Integrative Medicine, Nanjing University of Chinese Medicine, Nanjing 210046;

${ }^{2}$ Department of Clinical Laboratory, First Affiliated Hospital of Nanjing University of Chinese Medicine,

Nanjing 210029, P.R. China

Received August 13, 2012; Accepted November 9, 2012

DOI: $10.3892 / 01.2012 .1041$

\begin{abstract}
Epidermal growth factor (EGF) is critical in cancer progression and various genotypes of the EGF gene have been reported to be correlated with susceptibility to gastric cancer; however, the results are unclear. The aim of this study was to explore the associations of functional EGF gene polymorphisms and risk of gastric cancer in a Chinese population. We genotyped seven functional single-nucleotide polymorphisms (SNPs) of the EGF gene [rs3756261, rs11568835 and rs4444903 in the promoter region; rs11568943, rs2237051 and rs11569017 in the non-synonymous exon region and rs3733625 in the $3^{\prime}$ untranslated region (UTR)] in a hospitalbased case-control study, including 387 gastric cancer cases and 392 healthy controls by polymerase chain reactionligation detection reaction (PCR-LDR) methods. We found that individuals carrying the AG/GG genotype of rs2237051 (Ile to Met) in the exon region had an increased risk of gastric cancer (adjusted OR=1.577; 95\% CI=1.163-2.138), compared with the wild-type homozygous AA genotype. The heterozygous AG/GG genotype of rs3733625 in the 3'UTR demonstrated a protective effect (adjusted OR=0.690; 95\% CI=0.501-0.951), compared with the homozygous AA genotype. In addition, the effects of the two SNPs were more evident in intestinal gastric cancer $(\mathrm{P}<0.05)$; however, this was not case for the diffuse type $(\mathrm{P}>0.05)$. These findings indicate that a change in amino acids from isoleucine to methionine of rs2237051 and rs3733625 in the 3'UTR may contribute to the etiology of intestinal gastric cancer in the Chinese population.
\end{abstract}

Correspondence to: Professor Zhen Zhan, Department of Chinese and Western Integrative Medicine, Nanjing University of Chinese Medicine, 138 Xianlin Dadao, Jiangsu 210046, Nanjing, P.R. China E-mail: zhanzhan5607@sina.com

${ }^{*}$ Contributed equally

Key words: epidermal growth factor polymorphisms, gastric cancer, Chinese population, molecular epidemiology

\section{Introduction}

Gastric cancer remains the world's second most common malignancy, accounting for a large proportion of cancer cases in Asia, Latin America and certain countries in Europe (1). However, the etiology of gastric cancer is not well-understood. Epidemiological studies have suggested that environmental exposures, including a salty diet, tobacco smoking and Helicobacter pylori infection, have an effect on the development of gastric cancer $(2,3)$. In recent years, accumulating evidence suggests that genetic alternation may play an important role in gastric carcinogenesis.

Epidermal growth factor (EGF), first isolated in 1962, has a number of biological functions. When binding with EGFreceptor (EGFR), EGF activates multiple signaling pathways, regulating cell proliferation and differentiation of epithelial cells. Its expression is upregulated during liver regeneration and its overexpression in esophageal $(4,5)$ and gastric cancer $(6,7)$ has been shown to be involved in tumor invasion and poor prognosis. The EGF gene contains an atypical TATA box, polypurine-rich motifs and consensus binding sequences for a number of transcription factors, including nuclear factorkappa B (NF-kB), gastrin (GAS), activator protein (AP)-1, specificity protein (Sp)-1 and CCAAT/enhancer binding protein (C/EBP) (8). Common genetic variants in the EGF functional region [promoter, exons and 3 ' untranslated region (UTR)] may contribute to the inter-individual differences of EGF expression and subsequently disease susceptibility (9). Previous studies have reported that the EGF +61 (A/G) singlenucleotide polymorphism (SNP) in the 5'UTR of the EGF gene (SNP rs444903) is associated with various carcinomas, including gastric cancer (10-13). However, other research did not support the positive results on gastric cancer (14). Since the single locus may not represent the functional region of the gene, it is biologically possible that other functional variants may also be involved in gastric cancer susceptibility. We selected six functional SNPs of the EGF gene besides +61 A/G (rs3756261, rs11568835 and rs4444903 in the promoter region; rs11568943, rs2237051 and rs11569017 in the non-synonymous exon region and rs3733625 in the 3'-UTR region), with a minor allele frequency $>0.05$ in a Chinese population. To evaluate the effects of these EGF functional SNPs in gastric cancer 


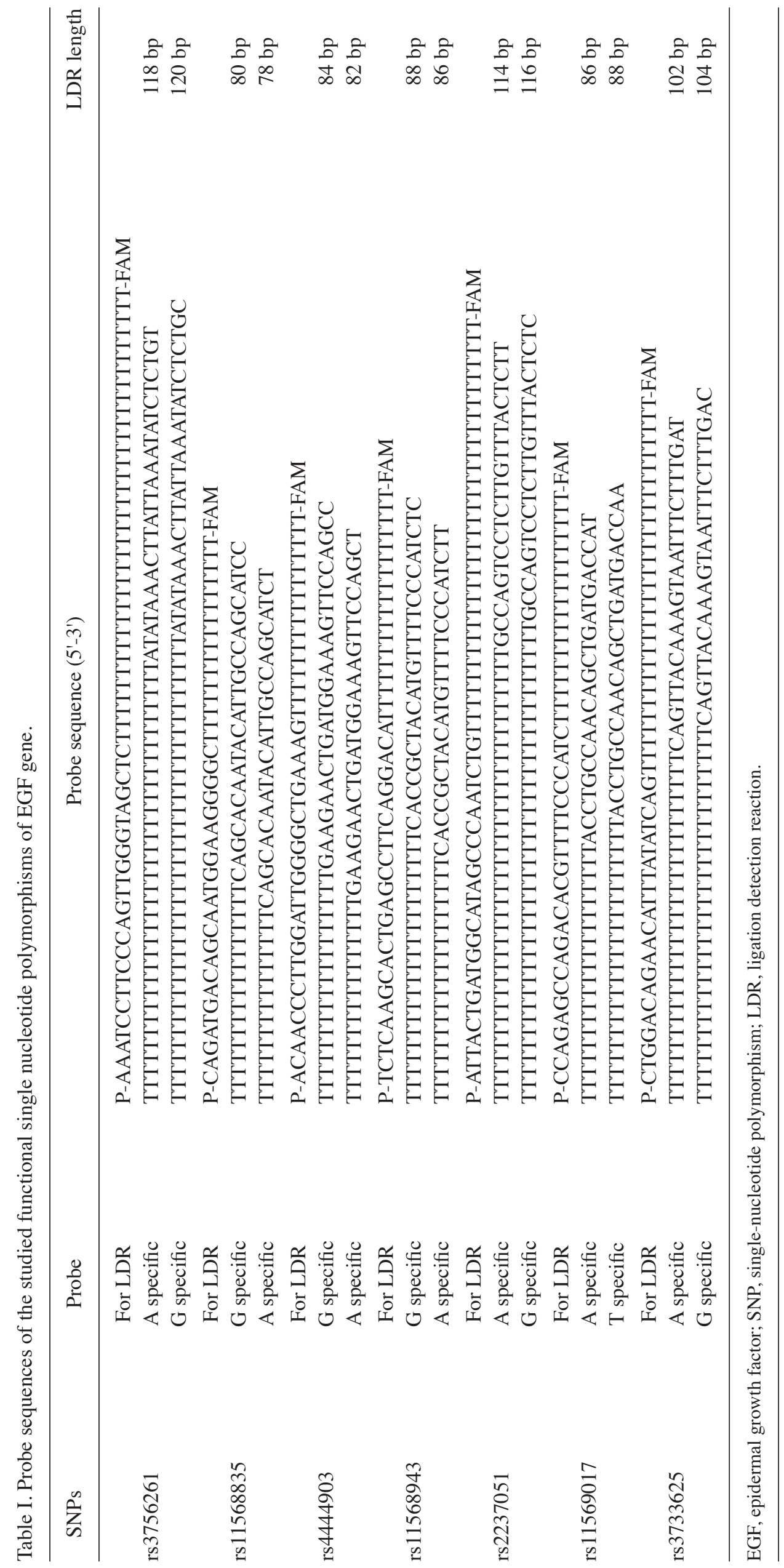


susceptibility, we conducted genotyping analyses for these SNPs in 387 gastric cancer cases and 392 cancer-free controls in a Chinese population.

\section{Materials and methods}

Study subjects. This study was approved by the institutional review board of Jiangsu Province Hospital of Chinese Medicine. All subjects were genetically unrelated Han Chinese. A total of 387 gastric cancer patients and 392 cancer-free healthy controls were consecutively recruited from January 2008 to July 2010 in Nanjing city, Jiangsu province, eastern China. The criteria for the recruitment of gastric cancer cases included: a) self-reported Han Chinese; b) local residents for at least 5 years; c) newly histopathologically diagnosed with primary gastric cancer; d) without a previous malignant tumor in any other organ and e) without any antitumor therapy prior to recruitment, including chemotherapy and radiotherapy. Any patients who met the above criteria and consented to participate in the study were included. As a result, a total of 400 gastric cancer cases were included in this study with a response rate of $96.8 \%$. The 400 cancer-free controls were collected from hospital visitors who came to the health examination clinic for an annual check-up at the same hospital during the same period. According to the criterion of Lauren's classification, gastric cancer cases were divided into two subgroups, intestinal type and diffuse type and those with mixed type or not available for classification were denoted with unclassified. A standard questionnaire was administered by trained interviewers to obtain information on demographic data and related risk factors, including tobacco smoking and alcohol consumption. After signing an informed consent form, a 3-5 ml venous blood sample was collected from each subject. A total of 387 gastric cancer cases and 392 unrelated healthy controls were included in this study. Individuals who smoked at least one cigarette per day for over 1 year were considered as smokers and those that had three or more alcohol drinks a week for over 6 months were defined as alcohol drinkers (15-16).

DNA extraction and genotyping. Venous blood $(3-5 \mathrm{ml})$ was collected from each subject and AxyPrep-96 kit (Axygen, CA, USA) was applied to extract genomic DNA. Polymerase chain reaction-ligation detection reaction (PCR-LDR) methods were used to perform the genotyping. The PCR primers for the seven loci (rs3756261, rs11568835, rs4444903, rs11568943, rs2237051, rs11569017 and rs3733625) were 5'-GCAGATGCTATGGCTGATGA-3' (forward), 5'-GAAGTGTGATCTGCCCACCT-3' (reverse) for rs3756261; 5'-GCAAACCTTTTTCCAACCAA-3' (forward), 5'-AACCTGCACTGACTCTTCGAG-3' (reverse) for rs11568835; 5'-CATTTGCAAACAGAGGCTCA-3' (forward), 5'-TGCTCTGGCTGACTTCACTG-3' (reverse) for rs4444903; 5'-TCCACGCAATGTGTCTGAAT-3' (forward), 5'-TCAATTTTTAGATGTCACTGAGCAA-3' (reverse) for rs11568943; 5'-TTCAAGCCTTGTCCTTTCGT-3' (forward), 5'-GTTTTGCCAATGGATGAACC-3' (reverse) for rs2237051; 5'-TGCAAAAAGAGGCTTGGAAC-3' (forward), 5'-TTCAAAATCAGCAAAAGCATAAA-3' (reverse) for rs11569017; 5'-CACGCCAATGAGGAGTTAAA-3' (forward),
Table II. Distribution of selected variables in gastric cancer cases and controls.

\begin{tabular}{lccc}
\hline Variable & GC cases & Controls & P-value \\
\hline Number & 387 & 392 & \\
Age (years) & $59.37 \pm 13.22$ & $50.63 \pm 11.74$ & $0.001^{\mathrm{a}}$ \\
Gender & & & $0.005^{\mathrm{b}}$ \\
Male & $271(70.0 \%)$ & $237(60.5 \%)$ & \\
Female & $116(30.0 \%)$ & $155(39.5 \%)$ & \\
Smoker & & & $0.001^{\mathrm{b}}$ \\
No & $204(52.7 \%)$ & $265(67.6 \%)$ & \\
Yes & $183(47.3 \%)$ & $127(32.4 \%)$ & \\
Drinker & & & $0.001^{\mathrm{b}}$ \\
No & $274(70.8 \%)$ & $324(82.7 \%)$ & \\
Yes & $113(29.2 \%)$ & $68(17.3 \%)$ & \\
Histological types & & & \\
Intestinal type & $280(72.3 \%)$ & & \\
Diffuse type & $58(15.0 \%)$ & & \\
Unclassified & $49(12.7 \%)$ & & \\
\hline
\end{tabular}

${ }^{a}$ Non-parametric test. ${ }^{b}$ Two-sided test. ${ }^{\text {Including }} 15$ mixed histological types and 34 unknown. GC, gastric cancer.

5'-CAAATTGGGACAACAGTGCTT-3' (reverse) for rs3733625. PCR was conducted on the ABI 9600 (Applied Biosystems, Foster City, CA, USA) in a system with a total volume of $15 \mu \mathrm{l}$, containing $1 \mu \mathrm{l}$ genomic DNA, $1.5 \mu \mathrm{l} 10 \mathrm{X}$ PCR buffer, $0.13 \mu \mathrm{M}$ each primer, $0.2 \mathrm{mM}$ deoxyribonucleotide triphosphate (dNTP), $0.25 \mu 1$ Taq DNA polymerase (Qiagen $\mathrm{GmbH}$, Hilden, Germany) and $7.5 \mu \mathrm{l} \mathrm{H}_{2} \mathrm{O}$. The cycling parameters were: $94^{\circ} \mathrm{C}$ for $1 \mathrm{~min} ; 35$ cycles at $94^{\circ} \mathrm{C}$ for $10 \mathrm{sec}$, $56^{\circ} \mathrm{C}$ for $20 \mathrm{sec}, 72^{\circ} \mathrm{C}$ for $40 \mathrm{sec}$ and a final extension step at $72^{\circ} \mathrm{C}$ for $3 \mathrm{~min}$. Probe sequences are shown in Table I. The probes for LDR have common phosphorylated 5 '-end and 6-carboxyfluorescein (FAM) labeled 3'-end. For each PCR product, the ligation reaction was performed in a final volume of $10 \mu \mathrm{l}$, including $2 \mu \mathrm{l}$ PCR product, $1 \mu \mathrm{l} 10 \mathrm{X}$ Taq DNA ligase buffer, $0.02 \mu \mathrm{M}$ probe mixture, 5 units Taq DNA ligase (New England Biolabs, Beverly, MA, USA) and $6 \mu 1 \mathrm{H}_{2} \mathrm{O}$. The LDR parameters were as follows: 25 cycles at $94^{\circ} \mathrm{C}$ for $30 \mathrm{sec}$ and $55^{\circ} \mathrm{C}$ for $4 \mathrm{~min}$. The LDR reaction products were analyzed on ABI 377 DNA sequencer (Applied Biosystems). To confirm the accuracy of the PCR-LDR genotyping method, 5\% samples were randomly selected for direct DNA sequencing for confirmation. PCR-LDR genotyping showed $100 \%$ concordance to direct DNA sequencing of the randomly selected PCR products.

Statistical analyses. The Hardy-Weinberg equilibrium was tested by a goodness-of-fit Chi-square test to compare the observed genotype frequencies with expected ones in the control group. Differences in the distribution of demographic characteristics, selected variables and genotypes of the EGF variants between the gastric cancer cases and controls were evaluated using the two-sided Chi-square test. Logistic regres- 
Table III. EGF gene polymorphisms on the risk of gastric cancer.

\begin{tabular}{|c|c|c|c|c|}
\hline Genotype & Gastric cancer cases & Controls & Crude OR (95\% CI) & Adjusted OR (95\% CI) \\
\hline Number & 387 & 392 & & \\
\hline rs 3756261 & 383 & 389 & & \\
\hline AA & 236 & 222 & 1.00 & 1.00 \\
\hline $\mathrm{AG}$ & 128 & 143 & $0.842(0.623-1.137)$ & $0.763(0.552-1.054)$ \\
\hline GG & 19 & 24 & $0.745(0.397-1.397)$ & $0.629(0.323-1.225)$ \\
\hline $\mathrm{AG} / \mathrm{GG}$ & 147 & 167 & $0.828(0.621-1.104)$ & $0.742(0.545-1.012)$ \\
\hline rs 11568835 & 366 & 368 & & \\
\hline GG & 269 & 269 & 1.00 & 1.00 \\
\hline GA & 89 & 92 & $0.967(0.691-1.355)$ & $1.014(0.708-1.452)$ \\
\hline AA & 8 & 7 & $1.143(0.409-3.196)$ & $1.090(0.362-3.275)$ \\
\hline GA/AA & 97 & 99 & $0.980(0.706-1.359)$ & $1.019(0.719-1.445)$ \\
\hline rs4444903 & 375 & 383 & & \\
\hline GG & 177 & 204 & 1.00 & 1.00 \\
\hline GA & 166 & 142 & $1.347(0.997-1.821)$ & $1.325(0.960-1.829)$ \\
\hline AA & 32 & 37 & $0.997(0.596-1.667)$ & $1.090(0.630-1.883)$ \\
\hline GA/AA & 198 & 179 & $1.275(0.958-1.696)$ & $1.278(0.942-1.734)$ \\
\hline rs 11568943 & 384 & 390 & & \\
\hline GG & 227 & 220 & 1.00 & 1.00 \\
\hline GA & 137 & 144 & $0.922(0.684-1.243)$ & $0.890(0.647-1.225)$ \\
\hline AA & 20 & 26 & $0.746(0.404-1.374)$ & $0.783(0.408-1.502)$ \\
\hline GA/AA & 157 & 170 & $0.895(0.673-1.191)$ & $0.874(0.644-1.186)$ \\
\hline rs2237051 & 384 & 387 & & \\
\hline AA & 162 & 206 & 1.00 & 1.00 \\
\hline $\mathrm{AG}$ & 193 & 150 & $1.636(1.216-2.201)$ & $1.656(1.205-2.274)$ \\
\hline GG & 29 & 31 & $1.190(0.689-2.055)$ & $1.202(0.672-2.150)$ \\
\hline $\mathrm{AG} / \mathrm{GG}$ & 222 & 181 & $1.560(1.174-2.073)$ & $1.577(1.163-2.138)$ \\
\hline rs11569017 & 365 & 381 & & \\
\hline AA & 225 & 222 & 1.00 & 1.00 \\
\hline AT & 123 & 135 & $0.899(0.662-1.222)$ & 0.798 (0.574-1.109) \\
\hline $\mathrm{TT}$ & 17 & 24 & $0.699(0.365-1.337)$ & $0.683(0.343-1.363)$ \\
\hline AT/TT & 140 & 159 & $0.869(0.648-1.165)$ & $0.781(0.571-1.070)$ \\
\hline rs3733625 & 384 & 388 & & \\
\hline AA & 267 & 243 & 1.00 & 1.00 \\
\hline $\mathrm{AG}$ & 106 & 127 & $0.760(0.557-1.037)$ & $0.714(0.512-0.996)$ \\
\hline GG & 11 & 18 & $0.556(0.258-1.201)$ & $0.518(0.227-1.182)$ \\
\hline $\mathrm{AG} / \mathrm{GG}$ & 117 & 145 & $0.734(0.544-0.991)$ & $0.690(0.501-0.951)$ \\
\hline
\end{tabular}

${ }^{a}$ Adjusted by age, gender, smoking and drinking status. EGF, epidermal growth factor; OR, odds ratio; CI, confidence interval.

sion analysis was employed to estimate crude and adjusted odds ratios (ORs) and 95\% confidence intervals (CIs) for the association between EGF genotypes and risk of gastric cancer. Analyses were performed using the software SPSS 16.0 (SPSS Inc., Chicago, IL, USA).

\section{Results}

Characteristics of study subjects. Gastric cancer cases (387 subjects) and cancer-free controls (392 subjects) were enrolled in our study. The age range for the participants included in the study was 19 to 90 years and the mean ages for the patients and controls were $59.37 \pm 13.22$ and $50.63 \pm 11.74$ years, respectively. The percentages of male and female participants were $70.0 \%$ and $30.0 \%$ in the gastric cancer group and $60.5 \%$ and $39.5 \%$ in the control group, respectively. The percentages of smokers in the gastric cancer group and the healthy control group were $47.3 \%$ and $32.4 \%$, respectively, while the percentages of drinkers were $29.2 \%$ and $17.3 \%$, respectively. Of the 387 gastric cancer cases included, $353(91.2 \%)$ patients' patho- 
Table IV. Stratified analyses between rs 2237051 and rs3733625 polymorphism and gastric cancer risk.

\begin{tabular}{|c|c|c|c|c|c|c|c|c|c|c|c|c|}
\hline \multirow{3}{*}{ Variable } & \multicolumn{6}{|c|}{ rs2237051 } & \multicolumn{6}{|c|}{ rs 3733625} \\
\hline & \multicolumn{2}{|c|}{ GC cases } & \multicolumn{2}{|c|}{ Controls } & \multicolumn{2}{|c|}{${ }^{\mathrm{a} A d j u s t e d ~ O R ~(95 \% C I) ~}$} & \multicolumn{2}{|c|}{ GC cases } & \multicolumn{2}{|c|}{ Controls } & \multicolumn{2}{|c|}{${ }^{\mathrm{a}}$ Adjusted OR $(95 \% \mathrm{CI})$} \\
\hline & AA & AG/GG & AA & $\mathrm{AG} / \mathrm{GG}$ & AA & AG/GG & AA & $\mathrm{AG} / \mathrm{GG}$ & AA & $\mathrm{AG} / \mathrm{GG}$ & AA & AG/GG \\
\hline \multicolumn{13}{|c|}{ Age (years) } \\
\hline$\leq 50$ & 32 & 45 & 106 & 91 & 1.00 & $1.64(0.96-2.79)$ & 51 & 26 & 129 & 71 & 1.00 & $0.93(0.53-1.61)$ \\
\hline$>50$ & 126 & 172 & 100 & 89 & 1.00 & $1.53(1.06-2.21)$ & 211 & 87 & 113 & 74 & 1.00 & $0.63(0.43-0.93)$ \\
\hline \multicolumn{13}{|l|}{ Gender } \\
\hline Male & 107 & 161 & 126 & 108 & 1.00 & $1.76(1.23-2.50)$ & 190 & 78 & 144 & 92 & 1.00 & $0.64(0.44-0.93)$ \\
\hline Female & 55 & 61 & 80 & 73 & 1.00 & $1.22(0.75-1.97)$ & 77 & 39 & 99 & 53 & 1.00 & $0.95(0.57-1.58)$ \\
\hline \multicolumn{13}{|l|}{ Smoker } \\
\hline No & 96 & 107 & 136 & 126 & 1.00 & $1.20(0.83-1.74)$ & 139 & 64 & 164 & 98 & 1.00 & $0.77(0.52-1.14)$ \\
\hline Yes & 66 & 115 & 70 & 55 & 1.00 & $2.22(1.39-3.53)$ & 128 & 53 & 79 & 47 & 1.00 & $0.70(0.43-1.13)$ \\
\hline \multicolumn{13}{|l|}{ Drinker } \\
\hline No & 117 & 154 & 163 & 156 & 1.00 & $1.38(0.99-1.90)$ & 189 & 82 & 207 & 114 & 1.00 & $0.79(0.56-1.11)$ \\
\hline Yes & 45 & 68 & 43 & 25 & 1.00 & $2.60(1.40-4.83)$ & 78 & 35 & 36 & 31 & 1.00 & $0.52(0.28-0.97)$ \\
\hline
\end{tabular}

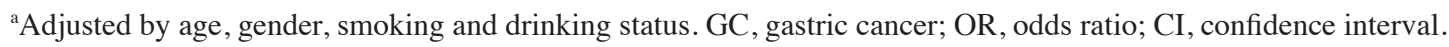

logical tissues were available for histological type dividing (58 diffuse gastric cancer cases, 280 intestinal gastric cancer cases and 15 gastric cancer cases with mixed histological type) according to the Lauren's classification. Meanwhile, 34 (8.8\%) patients' pathological tissues were not available for classification (Table II).

Genotyping distribution and risk of gastric cancer. The seven polymorphisms of EGF genotype distribution in the gastric cancer cases and controls are shown in Table III. The observed genotype distributions for all polymorphisms were in Hardy-Weinberg equilibrium in the controls $(\mathrm{P}=0.879$ for rs3756261, $\mathrm{P}=0.789$ for $\mathrm{rs} 11568835, \mathrm{P}=0.099$ for $\mathrm{rs} 4444903$, $\mathrm{P}=0.711$ for $\mathrm{rs} 11568943, \mathrm{P}=0.615$ for $\mathrm{rs} 2237051, \mathrm{P}=0.570$ for rs11569017 and $\mathrm{P}=0.788$ for rs3733625). rs2237051 (Ile to Met) in the exon region demonstrated a significant difference in the genotype distribution between gastric cancer cases and controls. Multiple logistic regression analysis revealed that individuals carrying the AG/GG genotype of rs2237051 (Ile to Met) in the exon region may have an increased risk of gastric cancer (adjusted $\mathrm{OR}=1.577$; 95\% CI=1.163-2.138), compared with the wild homozygous AA genotype. The heterozygous AG genotype of rs3733625 in the 3'UTR demonstrated a protective effect (adjusted OR $=0.714 ; 95 \%$ $\mathrm{CI}=0.512-0.996$ ), compared with homozygous AA genotype. However, we did not find an association between the $+61 \mathrm{~A} / \mathrm{G}$ (rs4444903) and gastric cancer. In the stratified analyses, we found that the risk effect of rs2237051 and the protective effect of rs3733625 was evident in subjects older than 50 year old, who were male and drinkers (Table IV). We further evaluated the genetic effects on gastric cancer according to the confirmed histological type (intestinal and diffuse type) and found that the risk effect of the $\mathrm{AG} / \mathrm{GG}$ genotype of rs2237051 and the protective effect of the AG/GG genotype of rs3733625 were more obvious in intestinal gastric cancer (adjusted $\mathrm{OR}=1.48,95 \% \mathrm{CI}=1.08-2.01$; adjusted $\mathrm{OR}=0.70$, $95 \% \mathrm{CI}=0.50-0.98$, respectively; $\mathrm{P}<0.05)$ but not in diffuse gastric cancer $(\mathrm{P}>0.05$; data not shown).

\section{Discussion}

In this case-control study, we investigated the seven functional polymorphisms of the EGF gene with the association of gastric cancer. We found that the variant genotype of EGF rs2237051 AG/GG is associated with an increased risk of intestinal gastric cancer and the variant genotype of EGF rs3733625 AG/GG is associated with a decreased risk of intestinal gastric cancer, compared with their wild-type homozygotes. In the stratified analyses, the effects of the two SNPs were more evident in those older than 50-years old who were male and drinkers.

The EGFR and the EGF-family of peptide growth factors play an important role in the development and progression of diverse carcinoma types, including gastric cancer $(10,13,17)$. Shahbazi et al (17) identified an SNP involving A to G transition at position 61 in the 5'UTR of the EGF gene (rs4444903) and the presence of the variant 61A allele led to decreased EGF production in peripheral blood mononuclear cells and decreased susceptibility of malignant melanoma. However, these results were not supported by later studies $(18,19)$. Recently, a number of studies have reported that the SNP rs4444903 is associated with various carcinomas, including gastric cancer (10-13). In the present study, we did not find an association between the rs4444903 variants and the risk of gastric cancer, which is consistent with the results reported by Goto et al (14). In addition, we found that SNP rs2237051 
(Ile to Met) in the exon region of EGF is associated with an increased risk of gastric cancer (particularly for the intestinal type) and another SNP rs3733625 in the 3'UTR is associated with a decreased risk of gastric cancer (particularly for the intestinal type). However, these results are initial and primitive. Amino acid alteration may influence the spatial conformation of the EGF protein and therefore alter the function of the gene. This may explain the correlation between the SNP rs2237051 of the EGF gene and risk of gastric cancer. Genetic variants in the 3'UTR may influence the stability of mRNA and therfore, the function of a gene. Notably, the two SNPs appeared to confer substantially greater effect in subsets of patients, namely males, elderly individuals and drinkers. Males and elderly individuals are associated with higher cumulative drinking exposure, thus drinking may result in the activation of carcinogenic pathways by the role of the two SNPs of the EGF gene; however, such a conclusion is premature. The two SNPs demonstrated an association with intestinal gastric cancer; however, not for the diffuse type, which may be due to the limited number of confirmed diffuse type gastric cancers. A larger sample size of diffuse type gastric cancer is required to validate this result.

Several limitations in our study need to be addressed: a) the function of the two polymorphisms of the EGF gene in the Chinese population have not been investigated; b) the sample size may limit the statistical power of our study, particularly for subgroup analysis of gastric cancer cases and stratified analysis. We adjusted possible confounding factors, including age, gender, smoking and drinking status, during the process of analysis; c) EGFR gene polymorphisms affecting EGFR activity were not examined.

In conclusion, our study shows that the two polymorphisms (rs2237051 and rs3733625) in the EGF gene may play a role in gastric cancer susceptibility and that drinking status may be a modulating factor. To explore the exact biological mechanism of the two SNPs, further functional studies and larger, well-designed studies, with ethnically diverse populations are required to confirm our findings.

\section{Acknowledgements}

This study was supported by the Priority Academic Program Development (PAPD) of Jiangsu Higher Education Institutions, Jiangsu Natural Science Foundation (BK2008461), the National Natural Science Foundation of China (30973715 and 81001502) and the Research Fund for the Doctoral Program of Higher Education of China (20103237110011).

\section{References}

1. Parkin DM, Pisani P and Ferlay J: Global cancer statistics. CA Cancer J Clin 49: 33-64, 1999.

2. Crew KD and Neugut AI: Epidemiology of upper gastrointestinal malignancies. Semin Oncol 31: 450-464, 2004.

3. Uemura N, Okamoto S, Yamamoto S, et al: Helicobacter pylori infection and the development of gastric cancer. N Engl J Med 345: 784-789, 2001.

4. Lu SH, Hsieh LL, Luo FC and Weinstein IB: Amplification of the EGF receptor and c-myc genes in human esophageal cancer. Int J Cancer 42: 502-505, 1988.

5. Stoscheck CM and King LE: Role of epidermal growth factor in carcinogenesis. Cancer Research 46:1030-1037, 1986.

6. Yasui W, Hata J, Yokozaki H, et al: Interaction between epidermal growth factor and its receptor in progression of human gastric carcinoma. Int J Cancer 41: 211-217, 1988.

7. Tokunaga A, Onda M, Okuda T, et al: Clinical significance of epidermal growth factor (EGF), EGF receptor, and c-erbB-2 in human gastric cancer. Cancer 75: 1418-1425, 1995.

8. Fenton SE, Groce NS and Lee DC: Characterization of the mouse epidermal growth factor promoter and 5'-flanking region. Role for an atypical TATA sequence. J Biol Chem 271: 30870-30878, 1996.

9. Mullhaupt B, Feren A, Jones A and Fodor E: DNA sequence and functional characterization of the human and rat epidermal growth factor promoter: regulation by cell growth. Gene 50: 191-200, 2000.

10. Zhu J, Meng X, Yan F, et al: A functional epidermal growth factor (EGF) polymorphism, EGF serum levels and renal cell carcinoma risk in a Chinese population. J Hum Genet 55: 236-240, 2010.

11. Chen K, Wei Y, Yang $\mathrm{H}$ and $\mathrm{Li} \mathrm{B}$ : Epidermal growth factor +61 G/A polymorphism and the risk of hepatocellular carcinoma in a Chinese population. Genet Test Mol Biomarkers 15: 251-255, 2011.

12. Amend KL, Elder JT, Tomsho LP, et al: EGF gene polymorphism and the risk of incident primary melanoma. Cancer Res 64: 2668-2672, 2004.

13. Hamai Y, Matsumura S, Matsusaki K, et al: A single nucleotide polymorphism in the 5' untranslated region of the EGF gene is associated with occurrence and malignant progression of gastric cancer. Pathobiology 72: 133-138, 2005.

14. Goto Y, Ando T, Goto H and Hamajima N: No association between EGF gene polymorphism and gastric cancer. Cancer Epidemiol Biomarkers Prev 14: 2454-2456, 2005.

15. Shen H, Xu Y, Qian Y, et al: Polymorphisms of the DNA repair gene XRCC1 and risk of gastric cancer in a Chinese population. Int J Cancer 88: 601-606, 2000.

16. Shen H, Xu Y,Zheng Y, et al: Polymorphisms of 5,10-methylenetetrahydrofolate reductase and risk of gastric cancer in a Chinese population: a case-control study. Int J Cancer 95: 332-336, 2001.

17. Shahbazi M, Pravica V, Nasreen N, et al: Association between functional polymorphism in EGF gene and malignant melanoma. Lancet 359: 397-401, 2002.

18. McCarron SL, Bateman AC, Theaker JM and Howell WM: EGF +61 gene polymorphism and susceptibility to and prognostic markers in cutaneous malignant melanoma. Int J Cancer 107: 673-675, 2003.

19. Randerson-Moor JA, Gaut R, Turner F, et al: The relationship between the epidermal growth factor (EGF) 5'UTR variant A61G and melanoma/nevus susceptibility. J Invest Dermatol 123: 755-759, 2004 